\title{
MINIREVIEW
}

\section{Sometimes one just isn't enough: do vertebrates contain an H2A.Z hyper-variant?}

\author{
Monika Mehta", Hyun-Soo Kim" and Michael-Christopher Keogh* \\ See research articles http://www.biomedcentral.com/1741-7007/7/86 and http://www.biomedcentral.com/1471-2148/9/31
}

\begin{abstract}
How much functional specialization can one component histone confer on a single nucleosome? The histone variant $\mathrm{H} 2 \mathrm{~A}$.Z seems to be an extreme example. Genome-wide distribution maps show non-random (and evolutionarily conserved) patterns, with localized enrichment or depletion giving a tantalizing suggestion of function. Multiple posttranslational modifications on the protein indicate further regulation. An additional layer of complexity has now been uncovered: the vertebrate form is actually encoded by two non-allelic genes that differ by expression pattern and three amino acids.
\end{abstract}

All species have to perform a balancing act with their genome: cram it into the cell (in the case of eukaryotes, a small part of that cell: for example, about $2 \mathrm{~m}$ of human DNA in a nucleus of about $5 \mu \mathrm{m}$ diameter) yet make the appropriate regions readily available for replication and expression (and repair if something goes wrong). Eukaryotes achieve this feat by wrapping their DNA into chromatin, a highly ordered complex with a simple repeating unit of about 146 bp DNA plus eight histone proteins, termed the nucleosome. This beads-on-a-string array is further assembled into a variety of higher-order structures all the way up to the metaphase chromosome. The chromatin field exploded with the discovery that the monotonous-looking nucleosomes are actually hugely variable, with post-translational modifications of numerous residues on the major histones, their substitution for histone variants, and even post-translational modifications of the variants. Furthermore, the highly

\footnotetext{
These authors contributed equally to this work.

*Correspondence: michael.keogh@einstein.yu.edu

Department of Cell Biology, Albert Einstein College of Medicine, New York, NY 10461, USA
}

regulated use of these marks serves to distinguish regions of DNA (such as promoters, centromeres or damaged DNA) to the appropriate enzymatic machineries.

Histone variants are non-allelic isoforms of the canonical histones that can be assembled into nucleosomes in their place, and are thought thereby to provide the basis for regulation of biological processes that require local access to DNA. In contrast to the S-phasecoupled synthesis of the major histones (timing availability to the peak demand of genome replication), variants are generally expressed throughout the cell cycle. Histone $\mathrm{H} 2 \mathrm{~A}$ has one of the largest variant families, and includes H2A.Z, a protein that is highly conserved across eukaryotes but differs considerably from the major H2A in each species (Figure 1) [1]. H2A.Z has been ascribed a large number of roles, including most recently suppressing antisense RNAs [2] and stabilizing the association of condensin with mitotic chromosomes [3]. Although we still have a poor understanding of how the variant mediates any specific function, it is likely that differential enrichment at specific locations and distinct post-translational modifications contribute. H2A.Z at the inactive $\mathrm{X}$ chromosome of mammalian female cells is monoubiquitinated [4], that in budding yeast (Saccharomyces cerevisiae) is sumoylated [5], and in all tested species it is subject to multiple amino-terminal acetylations (Figure 1), primarily by the Kat 5 family of acetyltransferases $[1,3]$. Mutation of the $S$. cerevisiae sumoylation sites impairs movement of DNA double-strand breaks to the nuclear periphery [5], whereas an unacetylatable allele in fission yeast (Schizosaccharomyces pombe) recapitulates many of the phenotypes of a complete deletion [3]. However, it is still unknown whether the effect of any of these modifications is direct (such as steric hindrance or charge modulation influencing the formation of higher-order structures) or indirect (such as generating sites for the recruitment of regulatory proteins).

\section{The impact of H2A.Z on individual nucleosomes}

The major route for H2A.Z into chromatin is via Swr1, the Snf2-family ATPase at the catalytic center of the SWR 


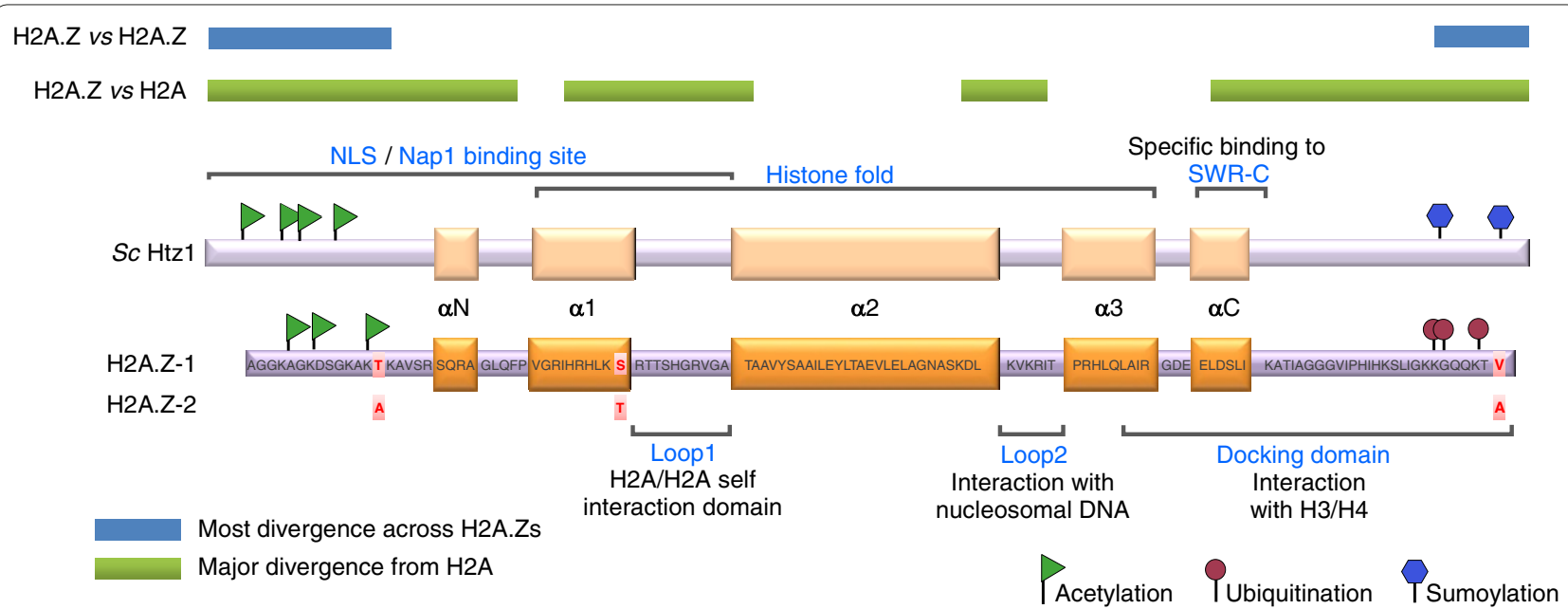

Figure 1. A simplified view of H2A.Z structure and post-translational modifications. Bars indicate the major regions of divergence between H2A.Z proteins across species (green) and between H2A.Z and H2A (blue). The relative location of the nuclear localization signal (NLS) and regions of S. cerevisiae (Sc) H2A.Z (called Htz1) that mediate contact with the Nap1 chaperone and the SWR-complex (SWR-C) ATPase complex are also shown. All H2A.Z post-translational modifications identified so far are on the relatively divergent amino and carboxyl termini, so it is unclear whether each specific modification is invariably used to regulate variant function across species. Addition of post-translational modifications generally depends on the SWR complex, indicating that each modification occurs after the variant is assembled into chromatin [3,5]. A major region of difference between H2A and H2A.Z is in the Loop 1 domain, which regulates interaction between the two H2A molecules in a nucleosome. This has led to the suggestion that nucleosome core particles can only be homotypic, containing either H2A or H2A.Z. However, hybrid nucleosomes containing H2A:H2B and H2A.Z:H2B dimers have been observed [6]. The sequence of human H2A.Z-1, including the three residues that differ in H2A.Z-2, is also indicated.

chromatin remodeling complex. This is certainly the case in S. cerevisiae (Swr1), S. pombe (Swr1), humans (SRCAP), Drosophila (Domino) and Arabidopsis (PIE1) [2,3,6-8]. The presence of H2A.Z in a nucleosome facilitates intramolecular folding to higher-order arrays, particularly 30 $\mathrm{nm}$ chromatin fibers, although these resist the formation of more highly condensed structures resulting from intermolecular association [6]. H2A.Z has also been reported to have a subtle destabilizing effect on the nucleosome in which it is incorporated, although this has been disputed. The apparent contradictions in the data can however be reconciled if the total histone composition of a nucleosome octamer is considered: variants seem to subtly alter nucleosome stability, so the order of stability is $\mathrm{H} 3$ / $\mathrm{H} 2 \mathrm{~A} . \mathrm{Z}=\mathrm{H} 3 / \mathrm{H} 2 \mathrm{~A}>\mathrm{H} 3.3 / \mathrm{H} 2 \mathrm{~A}>\mathrm{H} 3.3 / \mathrm{H} 2 \mathrm{~A} . \mathrm{Z}$ [9]. Furthermore, although it was originally predicted that H2A.Z was unlikely to form hybrid nucleosomes (Figure 1), both homotypic (containing two H2A.Z:H2B dimers) and heterotypic (containing H2A:H2B and H2A.Z:H2B dimers) forms have been observed, adding yet another level of structural (and possibly functional) heterogeneity $[6,10]$.

\section{Genome distribution maps of H2A.Z are suggestive of function}

High density maps of H2A.Z across genomes as diverse as S. cerevisiae [6], S. pombe [8], Arabidopsis thaliana [7] and Caenorhabditis elegans [2] show the variant to be widely but non-randomly distributed. In budding yeast
H2A.Z occupancy peaks in the single nucleosomes directly flanking $(-1 /+1)$ a nucleosome-free region over promoters, a pattern apparently induced by the nucleosome-free region itself [6]. Fission yeast, in contrast, shows enrichment in the +1 but not the -1 nucleosome around the nucleosome-free region [8]. The reason for (or outcome of) this difference is unknown, although in each organism enrichment depends on the SWR complex and is inversely correlated with transcriptional activity. H2A.Z has been linked to both transcriptional activation and repression at various genes, although consensus seems to be building towards a role in marking and/or poising promoters for expression. The presence of H2A.Z might commit local chromatin to a state competent for activation by other factors or, in higher eukaryotes, it might protect the region from inactivating DNA methylation [7]. Or it might be that the cell uses the inherent instability of H2A.Z-containing nucleosomes to regulate promoter accessibility.

\section{H2A.Z is found in 'hot' nucleosomes at promoters and euchromatin-heterochromatin boundaries}

Studies on the dynamics of replication-independent histone turnover in budding yeast suggest that H2A.Zcontaining nucleosomes have significantly higher turnover rates [6]. This 'hotness' of the nucleosomes around transcription start sites could aid promoter function by making it easier to expose these DNA elements to the 
transcriptional machinery. Such rapid flux might also abrogate the spread of a propagating domain (such as that of the budding yeast Sir2 deacetylase complex), which could explain how H2A.Z mediates a heterochromatin-euchromatin boundary function in this organism [6]. H2A.Z enrichment at the regions between euchromatin and heterochromatin is a feature also found in other organisms, despite the dramatic biochemical differences between the heterochromatin of budding yeast and that of many other species. In this manner the mammalian variant is a component of pericentric heterochromatin and flanks sites occupied by the insulator binding protein CTCF $[6,11]$.

\section{A vertebrate $\mathrm{H} 2 \mathrm{~A} . \mathrm{Z}$ hyper-variant}

As if the complexity described above wasn't enough, recent mass spectrometry analyses of chicken erythrocytes identified two forms of H2A.Z that differ by just three amino acids: H2A.Z-1 (previously H2A.Z) and H2A.Z-2 (previously H2A.F/Z or H2A.V) (Figure 1) [11]. These proteins are encoded by two non-allelic genes, which phylogenetic analyses indicate are present in all vertebrates and have a common origin early in chordate evolution [12]. Both isoforms are incorporated into chromatin, and both seem to be acetylated on the same three lysine residues within the amino terminus (Lys4, Lys7 and Lys11) to a similar degree (Figure 1) [11].

Why would vertebrates need two copies of a protein that differ by just three amino acids, whereas invertebrates do just fine with just one? Mouse studies ostensibly showing that H2A.Z is indispensable deleted only H2A.Z-1, indicating non-redundancy: that is, H2A.Z-2 cannot compensate. At this stage, we can only speculate about the relevance of each isoform. The three-aminoacid difference is not expected to have any major structural implication for nucleosomes [12]. The H2A.Z isoforms could have a differential affinity for various chaperones and/or deposition machineries, which could explain their subtly different chromatin occupancy patterns [11]. Although this might sound unlikely, there is a comparable precedent: three of the four amino acids that differ between histones H3.1 and H3.3 regulate the usage of the respective proteins in the replicationdependent and -independent deposition pathways [13]. However, the most important difference between H2A.Z-1 and H2A.Z-2 may be their highly divergent promoter sequences [11]. This opens up the possibility of dramatically different temporal and/or spatial expression patterns for the two isoforms. Indeed, preliminary studies suggest some differences in mRNA expression levels depending on the developmental stage of a variety of tissues [11].
Plants also have multiple H2A.Z isoforms. Three have been reported in A. thaliana - HTA8, HTA9 and HTA11 which share about $90 \%$ identity but have distinct expression patterns, with HTA9 alone being cell-cycleindependent [7]. PIE1 (the plant homolog of Swr1) interacts with all three variants, but not with H2A. Single knockouts have no distinct phenotypes, but double hta9/ hta11 knockouts show developmental abnormalities. It remains to be seen whether these variants of the variant have any tissue or developmental function [7]. However, the parallel with vertebrates is striking and may suggest that more complex organisms need more finely tuned chromatin than one H2A.Z can provide.

Published: 21 January 2010

\section{References}

1. Thambirajah AA, Ishibashi T, Ausio J: New developments in posttranslational modifications and functions of histone $\mathrm{H} 2 \mathrm{~A}$ variants. Biochem Cell Biol 2009, 87:7-17

2. Zofall M, Fischer T, Zhang K, Zhou M, Cui B, Veenstra TD, Grewal SI: Histone H2A.Z cooperates with RNAi and heterochromatin factors to suppress antisense RNAs. Nature 2009, 461:419-422.

3. Kim HS, Vanoosthuyse V, Fillingham J, Roguev A, Watt S, Kislinger T, Treyer A, Carpenter LR, Bennett CS, Emili A, Greenblatt JF, Hardwick KG, Krogan NJ, Bähler J, Keogh MC: An acetylated form of histone H2A.Z regulates chromosome architecture in Schizosaccharomyces pombe. Nat Struct Mol Biol 2009, 16:1286-1293

4. Sarcinella E, Zuzarte PC, Lau PN, Draker R, Cheung P: Monoubiquitylation of H2A.Z distinguishes its association with euchromatin or facultative heterochromatin. Mol Cell Bio/ 2007, 27:6457-6486.

5. Kalocsay M, Hiller NJ, Jentsch S: Chromosome wide Rad51 spreading and SUMO-H2A.Z-dependent chromosome fixation in response to a persistent DNA double-strand break. Mol Cell 2009, 33:335-343.

6. Zlatanova J, Thakar A: H2A.Z: view from the top. Structure 2008, 16:166-179.

7. March-Díaz R, Reyes JC: The beauty of being a variant: H2A.Z and the SWR1 complex in plants. Mol Plant 2009, 2:565-577.

8. Buchanan L, Durand-Dubief M, Roguev A, Sakalar C, Wilhelm B, Strålfors A, Shevchenko A, Aasland R, Shevchenko A, Ekwall K, Francis Stewart A: The Schizosaccharomyces pombe JmjC-protein, Msc1, prevents H2A.Z localization in centromeric and subtelomeric chromatin domains. PLOS Genetics 2009, 5:e1000726.

9. Jin C, Felsenfeld G: Nucleosome stability mediated by histone variants $\mathrm{H} 3.3$ and H2A.Z. Genes Dev 2007, 21:1519-1529.

10. Ishibashi T, Dryhurst D, Rose KL, Shabanowitz J, Hunt DF, Ausió J: Acetylation of vertebrate $\mathrm{H} 2 \mathrm{~A} . \mathrm{Z}$ and its effect on the structure of the nucleosome. Biochemistry 2009, 48:5007-5017.

11. Dryhurst D, Ishibashi T, Rose KL, Eirín-López JM, McDonald D, Silva-Moreno B, Veldhoen N, Helbing C, C, Hendzel MJ, Shabanowitz J, Hunt DF, Ausio J: Characterization of the histone H2A.Z-1 and H2A.Z-2 isoforms in vertebrates. BMC Biol 2009, 7:86

12. Eirín-López JM, González-Romero R, Dryhurst D, Ishibashi T, Ausió J: The evolutionary differentiation of two histone $H 2 A . Z$ variants in chordates (H2A.Z-1 and H2A.Z-2) is mediated by a stepwise mutation process that affects three amino acid residues. BMC Evol Biol 2009, 9:31

13. Ahmad K, Henikoff S: The histone variant H3.3 marks active chromatin by replication-independent nucleosome assembly. Mol Cell 2002, 9:1191-1200.

doi:10.1186/jbiol214

Cite this article as: Mehta M, et al:: Sometimes one just isn't enough: do vertebrates contain an H2A.Z hyper-variant? Journal of Biology 2010, 9:3. 